\title{
Relating CGC and TMD factorization frameworks beyond leading twist
}

\author{
Piotr Kotko*tł \\ Institute of Nuclear Physics PAN, Krakow (on leave) \\ AGH University of Science and Technology, Krakow \\ E-mail: piotr.kotko@fis.agh.edu.pl
}

It is known already for some time that the leading power limit of the Color Glass Condensate (CGC) expressions for dijet production can be identified with the (generalized) Transverse Momentum Dependent (TMD) factorization in the small $x$ regime. In the latter formalism, there are several TMD gluon distributions containing distinct Wilson line operators, and corresponding on-shell hard factors. There is a natural extension of this formalism, which generalizes the onshell hard factors to the off-shell case in a gauge invariant way - the so-called small-x improved TMD factorization (ITMD), which allows to study the kinematic regime beyond the back-to-back configuration, and thus beyond the leading twist. In this contribution we briefly recall the ITMD formalism, flash a recent phenomenology study of dijet production in proton-proton and protonlead collisions at the LHC, and - most importantly - we report on the recent proof of the ITMD approach from the CGC theory.

European Physical Society Conference on High Energy Physics - EPS-HEP2019 -

10-17 July, 2019

Ghent, Belgium

\footnotetext{
* Speaker.

${ }^{\dagger}$ In collaboration with Tolga Altinoluk and Renaud Boussarie.

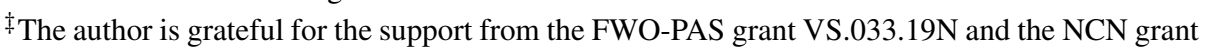
DEC-2017/27/B/ST2/01985.
} 


\section{Introduction}

One of the predictions of Quantum Chromodynamics (QCD) is that hadrons probed at very high energies reveal a dense partonic structure, with (mostly) gluons tightly packed within the hadron volume. In such regime, splitting of gluons due to further increase of the energy competes with the gluon recombination and leads to the so-called gluon saturation (see [1] for a comprehensive review of the field). A well-studied effective theory describing dynamics of such system is the Color Glass Condensate (CGC) (see eg. [2]). Within the conventional CGC theory one can describe various scattering processes off a dense target and its evolution with energy in the eikonal approximation. Each particle interacting with the color field of a target picks up a Wilson line in adequate color representation, where the gauge fields are functionals of the random color sources in the target. In the end, the result is averaged functionally over color sources with certain functional, which evolves with energy according to the Balitsky-JIMWLK (Balitsky-Jalilian-Marian-IancuMcLerran-Weigert-Leonidov-Kovner) equation (see also [1] and references therein).

While CGC theory is a comprehensive (though approximate) way of describing such processes, it becomes increasingly complicated when multiparticle color states are produced and tagged. Phenomenologically important example here is a production of forward jets in protonnucleus collisions (forward with respect to the proton beam). The expressions involve proliferated correlators of Wilson lines that characterize the nucleus. Though these objects are ultimately nonperturbative, their relation to the rather well studied Transverse Momentum Dependent (TMD) parton distributions (PDFs) (more precisely gluon distributions in the present context) is not obvious. In the past the correspondence was investigated in [3], focusing on dijet production and the leading twist limit, as this is the regime of the TMD factorization (see [4] for a comprehensive treatment of the TMD factorization). Further, a similar correspondence was shown for dijets plus a photon production process in [5], at leading twist. The gist of these investigations is the following. At leading twist, in the dilute-dense collisions, one has a proliferation of the TMD gluon distributions that correspond to the correlation (or back-to-back) limit of the CGC correlators. The hard factors appearing in the correlation limit turn out to be exactly the on-shell hard factors that must accompany the TMD gluon distributions.

In [6] a natural extension of [3] beyond the leading twist was proposed. The crucial element is the use of the off-shell gauge invariant hard matrix elements, instead of the on-shell ones accompanying the TMD gluon distributions. Such approach has been dubbed in the literature as the small-x Improved TMD factorization (ITMD). Note, that while this approach takes into account higher twist corrections, it neglects the multi-body operators for the TMD gluon distributions (multiparticle interactions). These multi-body operators are however present (in eikonal limit) in the CGC approach, but they are mixed with the twist corrections of the type that has been included in the ITMD. The clear correspondence of the ITMD approach and the CGC was obtained recently in [7]. Further, in [8] an investigation of the multi-body TMD operators in correspondence with the CGC has been initiated.

In this contribution, first we briefly recall the ITMD approach and show some recent phenomenological results (Sec. 2). Next, in Sec. 3, we report on the proof of the ITMD approach from the CGC theory which has been accomplished in [7]. 
Table 1: The hard factors and small $x$ TMD gluon distributions appearing in the ITMD factorization formula Eq. (2.1). The invariants are defined as follows: $\hat{s}=\left(p_{1}+p_{2}\right)^{2}, \bar{s}=\left(x_{A} p_{A}+k_{B}\right)^{2}, \hat{t}=\left(p_{1}-k_{A}\right)^{2}, \bar{t}=$ $\left(x_{A} p_{A}-p_{1}\right)^{2}, \hat{u}=\left(p_{1}-k_{B}\right)^{2}, \bar{u}=\left(x_{A} p_{A}-p_{2}\right)^{2}$.

$$
\begin{array}{cc}
K_{g q \rightarrow g q}^{(1)}=-\frac{\bar{u}\left(\bar{s}^{2}+\bar{u}^{2}\right)}{2 \bar{t} \hat{s}}\left(1+\frac{\bar{s} \hat{s}-\bar{t} \hat{t}}{N_{c}^{2} \bar{u} \hat{u}}\right) & \Phi_{g q \rightarrow g q}^{(1)}=\mathscr{F}_{q g}^{(1)} \\
K_{g q \rightarrow g q}^{(2)}=-\frac{C_{F}}{N_{c}} \frac{\bar{s}\left(\bar{s}^{2}+\bar{u}^{2}\right)}{\bar{t} \hat{t} \hat{u}} & \Phi_{g q \rightarrow g q}^{(2)}=\frac{1}{N_{c}^{2}-1}\left(-\mathscr{F}_{q g}^{(1)}+N_{c}^{2} \mathscr{F}_{q g}^{(2)}\right) \\
K_{g g \rightarrow q \bar{q}}^{(1)}=\frac{1}{2 N_{c}} \frac{\left(\bar{t}^{2}+\bar{u}^{2}\right)(\bar{u} \hat{u}+\bar{t} \hat{t})}{\bar{s} \hat{s} \hat{t} \hat{u}} & \Phi_{g g \rightarrow q \bar{q}}^{(1)}=\frac{1}{N_{c}^{2}-1}\left(N_{c}^{2} \mathscr{F}_{g g}^{(1)}-\mathscr{F}_{g g}^{(3)}\right) \\
K_{g g \rightarrow q \bar{q}}^{(2)}=\frac{1}{4 N_{c}^{2} C_{F}} \frac{\left(\bar{t}^{2}+\bar{u}^{2}\right)(\bar{u} \hat{u}+\bar{t} \hat{t}-\bar{s} \hat{s})}{\bar{s} \hat{s} \hat{u} \hat{u}} & \Phi_{g g \rightarrow q \bar{q}}^{(2)}=-N_{c}^{2} \mathscr{F}_{g g}^{(2)}+\mathscr{F}_{g g}^{(3)} \\
K_{g g \rightarrow g g}^{(1)}=\frac{N_{c}}{C_{F}} \frac{\left(\bar{s}^{4}+\bar{t}^{4}+\bar{u}^{4}\right)(\bar{u} \hat{u}+\bar{t} \hat{t})}{\bar{t} \hat{t} \bar{u} \hat{u} \bar{s} \hat{s}} & \Phi_{g g \rightarrow g g}^{(1)}=\frac{1}{2 N_{c}^{2}}\left(N_{c}^{2} \mathscr{F}_{g g}^{(1)}-2 \mathscr{F}_{g g}^{(3)}+\mathscr{F}_{g g}^{(4)}\right. \\
\left.+\mathscr{F}_{g g}^{(5)}+N_{c}^{2} \mathscr{F}_{g g}^{(6)}\right) & \begin{array}{c}
\Phi_{g g \rightarrow g g}= \\
N_{c}^{2}\left(N_{c}^{2} \mathscr{F}_{g g}^{(2)}-2 \mathscr{F}_{g g}^{(3)}+\mathscr{F}_{g g}^{(4)}\right. \\
\left.\mathscr{F}_{g g}^{(5)}+N_{c}^{2} \mathscr{F}_{g g}^{(6)}\right)
\end{array} \\
K_{g g \rightarrow g g}^{(2)}=-\frac{N_{c}}{2 C_{F}} \frac{\left(\bar{s}^{4}+\bar{t}^{4}+\bar{u}^{4}\right)(\bar{u} \hat{u}+\bar{t} \hat{t}-\bar{s} \hat{s})}{\bar{t} \hat{t} \hat{u} \hat{u} \hat{s} \hat{s}} &
\end{array}
$$

\section{Small-x Improved TMD factorization (ITMD)}

We consider production of dijets in proton-nucleus collisions, $p+A \rightarrow 2 j+X$. We assume the jets are forward, so that the nucleus is probed at very small longitudinal fractions $x_{A}$ of its momentum, while the parton from the proton is found in the large $x_{B}$ state. Thus the collision is of the dilute-dense type and the hybrid approach can be applied, i.e. the proton is described by the usual PDFs, while the nucleus needs a treatment adequate at the small- $x$ regime. Further, we assume that the jets give a hard scale $\mu$ of the order their average transverse momentum, $\mu \gg Q_{s} \gg \Lambda_{\mathrm{QCD}}$, where $Q_{s}$ is the saturation scale.

Following [6], the cross section for this process can be calculated by means of the following leading order factorization formula

$$
\frac{d \sigma^{p A \rightarrow 2 j+X}}{d^{2} q_{T} d^{2} k_{T} d y_{1} d y_{2}}=\frac{\alpha_{s}^{2}(\mu)}{\left(x_{A} x_{B} S\right)^{2}} \sum_{a, c, d} x_{B} f_{a / p}\left(x_{B}, \mu\right) \sum_{i=1}^{2} K_{a g^{*} \rightarrow c d}^{(i)}\left(q_{T}, k_{T} ; \mu\right) \Phi_{a g \rightarrow c d}^{(i)}\left(x_{\mathrm{A}}, k_{T}, \mu\right) .
$$

Above, $f_{a / p}$ is the collinear PDF for parton $a, K_{a g^{*} \rightarrow c d}^{(i)}$ are off-shell hard factors for partonic subprocess $a g \rightarrow c d$ (two per each sub-process due to two non-equivalent color flows), and $\Phi_{a g \rightarrow c d}^{(i)}$ are small- $x$ TMD gluon distributions for each hard factor. The sum over parton species must be obviously restricted so that $a g \rightarrow c d$ can be physically possible. The momentum conservation at the partonic level is $k_{A}+k_{B}=p_{1}+p_{2}$, where $k_{A}=x_{A} p_{A}+k_{T}$ is the momentum of a gluon probing the 
nucleus, $k_{B}=x_{B} p_{B}$ is the momentum of a parton probing the proton and $p_{1}, p_{2}$ are the momenta of jets. Here $p_{A}, p_{B}$ are momenta of the nucleus and the proton, respectively. Further, $\vec{q}_{T}=\vec{p}_{T 1}+\vec{p}_{T 1}$ is the transverse momentum imbalance for the jets and $y_{1}, y_{2}$ are the rapidities of the jets. The hard factors with off-shell gluons and the TMD gluon distributions are listed in Table 1 - see [6] and references therein for the methods to calculate them preserving the gauge invariance. The TMD gluon distributions are constructed as linear combinations of the basic distributions defined in terms of the gluonic bilocal operators off-the-light-cone. They are non-universal, i.e. the operator structure depends on the color flow in the hard process [9],[10]. For the present case we have [3]

$$
\begin{aligned}
& \mathscr{F}_{q g}^{(1)}=2 \int \frac{d \xi^{+} d^{2} \xi_{T}}{(2 \pi)^{3} p_{A}^{-}} e^{i x_{A} p_{A}^{-} \xi^{+}-i \vec{k}_{T} \cdot \vec{\xi}_{T}}\left\langle p_{A}\left|\operatorname{Tr}\left\{F(\xi) \mathscr{U}^{[-] \dagger} F(0) \mathscr{U}^{[+]}\right\}\right| p_{A}\right\rangle, \\
& \mathscr{F}_{q g}^{(2)}=2 \int \frac{d \xi^{+} d^{2} \xi_{T}}{(2 \pi)^{3} p_{A}^{-}} e^{i x_{A} p_{A}^{-} \xi^{+}-i \vec{k}_{T} \cdot \vec{\xi}_{T}}\left\langle p_{A}\left|\operatorname{Tr}\left\{F(\xi) \frac{\operatorname{Tr} \mathscr{U}^{[\square]}}{N_{c}} \mathscr{U}^{[+] \dagger} F(0) \mathscr{U}^{[+]}\right\}\right| p_{A}\right\rangle, \\
& \mathscr{F}_{g g}^{(1)}=2 \int \frac{d \xi^{+} d^{2} \xi_{T}}{(2 \pi)^{3} p_{A}^{-}} e^{i x_{A} p_{A}^{-} \xi^{+}-i \vec{k}_{T} \cdot \vec{\xi}_{T}}\left\langle p_{A}\left|\operatorname{Tr}\left\{F(\xi) \frac{\operatorname{Tr} \mathscr{U}^{[\square]}}{N_{c}} \mathscr{U}^{[-] \dagger} F(0) \mathscr{U}^{[+]}\right\}\right| p_{A}\right\rangle, \\
& \mathscr{F}_{g g}^{(2)}=2 \int \frac{d \xi^{+} d^{2} \xi_{T}}{(2 \pi)^{3} p_{A}^{-}} e^{i x_{A} p_{A}^{-} \xi^{+}-i \vec{k}_{T} \cdot \vec{\xi}_{T}} \frac{1}{N_{c}}\left\langle p_{A}\left|\operatorname{Tr}\left\{F(\xi) \mathscr{U}^{[\square] \dagger}\right\} \operatorname{Tr}\left\{F(0) \mathscr{U}^{[\square]}\right\}\right| p_{A}\right\rangle, \\
& \mathscr{F}_{g g}^{(3)}=2 \int \frac{d \xi^{+} d^{2} \xi_{T}}{(2 \pi)^{3} p_{A}^{-}} e^{i x_{A} p_{A}^{-} \xi^{+}-i \vec{k}_{T} \cdot \vec{\xi}_{T}}\left\langle p_{A}\left|\operatorname{Tr}\left\{F(\xi) \mathscr{U}^{[+] \dagger} F(0) \mathscr{U}^{[+]}\right\}\right| p_{A}\right\rangle, \\
& \mathscr{F}_{g g}^{(4)}=2 \int \frac{d \xi^{+} d^{2} \xi_{T}}{(2 \pi)^{3} p_{A}^{-}} e^{i x_{A} p_{A}^{-} \xi^{+}-i \vec{k}_{T} \cdot \vec{\xi}_{T}}\left\langle p_{A}\left|\operatorname{Tr}\left\{F(\xi) \mathscr{U}^{[-] \dagger} F(0) \mathscr{U}^{[-]}\right\}\right| p_{A}\right\rangle,
\end{aligned}
$$

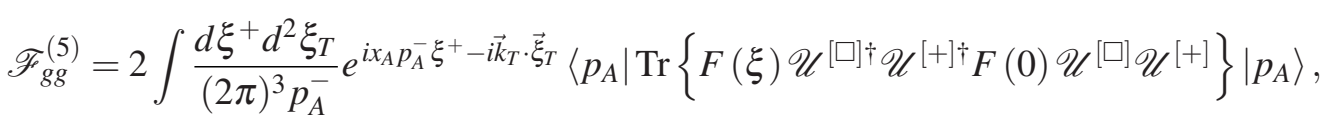

$$
\begin{aligned}
& \mathscr{F}_{g g}^{(6)}=2 \int \frac{d \xi^{+} d^{2} \xi_{T}}{(2 \pi)^{3} p_{A}^{-}} e^{i x_{A} p_{A}^{-} \xi^{+}-i \vec{k}_{T} \cdot \vec{\xi}_{T}}\left\langle p_{A}\left|\operatorname{Tr}\left\{F(\xi) \mathscr{U}^{[+] \dagger} F(0) \mathscr{U}^{[+]}\right\}\left(\frac{\operatorname{Tr} \mathscr{U}^{[\square]}}{N_{c}}\right)^{2}\right| p_{A}\right\rangle \text {, }
\end{aligned}
$$

where $F(\xi)=F_{a}^{-i}\left(\xi^{+}, \xi_{T}, 0\right) t^{a}$, with the following definitions of gauge links and loops:

$$
\mathscr{U}^{[ \pm]}=U\left(0, \pm \infty ; 0_{T}\right) U\left( \pm \infty, \xi^{+} ; \xi_{T}\right), \quad \mathscr{U}^{[\square]}=\mathscr{U}^{[+]} \mathscr{U}^{[-] \dagger}=\mathscr{U}^{[-]} \mathscr{U}^{[+] \dagger},
$$

where $U\left(a, b ; x_{T}\right)=\mathscr{P} \exp \left\{\operatorname{ig} \int_{a}^{b} d x^{+} A_{a}^{-}\left(x^{+}, x_{T}\right) t^{a}\right\}$. Above, we have neglected the transverse pieces of the gauge links as they give subleading contribution in the high energy limit.

Despite its complexity, the above factorization formula can be tested phenomenologically. The essential step is the determination of the apriori unknown TMD gluon distributions. So far it has been done basing on the correspondence with the CGC correlators. One way is to use the Gaussian approximation, large $N_{c}$ limit and the non-linear gluon distribution (equivalent to $F_{q g}^{(1)}$ ) of [11] fitted to HERA data, in order to obtain the rest distributions. This has been accomplished in [12]. Another way is to use the B-JIMWLK equation as has been done in [13], with however initial distribution not fitted to any data. Recently, the former method has been applied to calculate the azimuthal broadening in $p p$ and $p \mathrm{~Pb}$ collisions as measured by the ATLAS collaboration [14], showing importance of both saturation phenomenon and the Sudakov resummation [15]. The plot illustrating this result is shown in Fig. 1. 

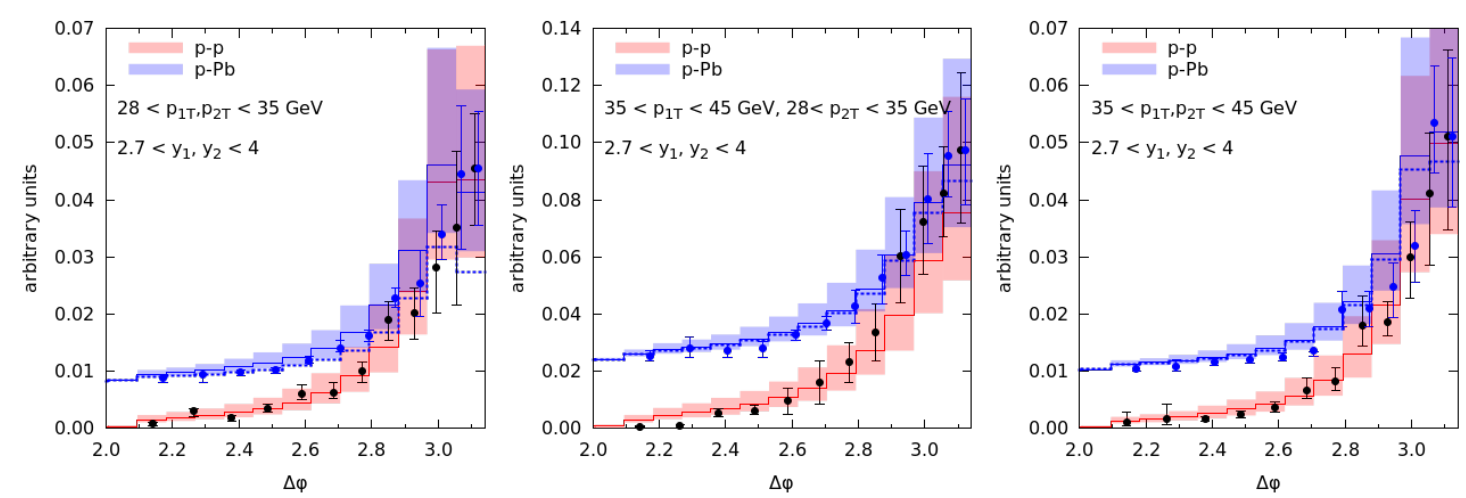

Figure 1: Azimuthal decorrelations (i.e. cross section as a function of the azimuthal angle between the jest) in $p \mathrm{~Pb}$ and $p p$ collisions, for different cuts on the jet transverse momenta, calculated using the ITMD formalism in [15] and compared with the experimental data [14]. The cross sections are presented in such a way that the values for $p \mathrm{~Pb}$ and $p p$ agree for the first bin, so that the broadening is apparent. See [15] for the details.

\section{Relation of the ITMD and CGC}

Before we sketch the derivation of the ITMD formula from the CGC formalism, as carried out in [7], let us make some initial remarks to set up the terminology.

First, it is convenient to work at the amplitude level. We consider a generic hard amplitude $\mathscr{H}_{i}\left(k_{T 1}, \ldots, k_{T i}\right)$ which exchanges $i$ gluons with the soft matrix element

$$
\mathscr{O}_{i}\left(k_{T 1}, \ldots, k_{T i}\right) \sim \text { F.T. }\left\langle p_{A}\left|F_{a_{1}}^{-k_{1}}\left(x_{1}\right) \ldots F_{a_{i}}^{-k_{i}}\left(x_{i}\right)\right| X\right\rangle,
$$

where F.T. stands for the Fourier transform. Above, we indicate only transverse momentum exchanged by each gluon, as a base for the power counting. Expanding the hard part in the transverse momenta, we obtain the following classification:

$$
\begin{aligned}
& \text { leading twist: } \quad \mathscr{A}_{L T}=\mathscr{H}_{1}(0) \otimes \mathscr{O}_{1}\left(k_{T 1}\right) \\
& \text { next-to-leading twist: } \quad \mathscr{A}_{N L T}=\vec{k}_{T 1} \cdot\left(\vec{\partial} \mathscr{H}_{1}\right)(0) \otimes \mathscr{O}_{1}\left(k_{T 1}\right) \quad \text { (kinematic twist) } \\
& +\mathscr{H}_{2}(0) \otimes \mathscr{O}_{2}\left(k_{T 1}, k_{T 2}\right) \quad \text { (genuine twist) }
\end{aligned}
$$

It turns out that the ITMD formula precisely resums all kinematic twists, while it neglects the genuine twists.

The demonstration of this fact consist of a few steps:

1. Obtaining a generic expression for the CGC amplitude to produce two partons, i.e. the splitting $0 \rightarrow 1+2$ in the presence of target color field:

$$
\begin{aligned}
\mathscr{A}=\delta\left(p_{1}^{+}+p_{2}^{+}-p_{0}^{+}\right) \int d^{2} b_{T} d^{2} r_{T} e^{-i\left(\vec{P}_{T} \cdot \vec{r}_{T}+\vec{k}_{T} \cdot \vec{b}_{T}\right)} \frac{r_{T}^{\mu}}{r_{T}^{2}} \\
\\
\left\{U^{R_{1}}\left(\vec{b}_{T}+\vec{z}_{T}\right) T^{R_{0}} U^{R_{2}}\left(\vec{b}_{T}-z \vec{r}_{T}\right)-U^{R_{1}}\left(\vec{b}_{T}\right) T^{R_{0}} U^{R_{2}}\left(\vec{b}_{T}\right)\right\} \Gamma_{\mu},
\end{aligned}
$$


where $p_{0}^{+}$is the longitudinal fraction of the parton from the dilute probe, $\vec{P}_{T}=\bar{z} \vec{p}_{T 1}-z \vec{p}_{T 2}$, $\vec{k}_{T}=\vec{p}_{T 1}+\vec{p}_{T 2}$, where $z=p_{2}^{+} / p_{0}^{+}$. The Wilson line is defined as

$$
U\left(x_{T}\right)=\mathscr{P} \exp \left\{i g \int_{-\infty}^{+\infty} d x^{+} A_{a}^{-}\left(x^{+}, x_{T}\right) t^{a}\right\}
$$

The $R_{0}, R_{1}, R_{2}$ are color representations of the incoming parton (originating in the dilute probe) and the two partons to which it splits - respectively. $\Gamma_{\mu}$ is the generic expression for the process dependent factorizable Dirac structure.

2. Taylor expansion in $r_{T}$. Since we want to have arbitrary $k_{T}$, as in the ITMD formalism, we perform an expansion in $r_{T}$, which is pushed towards small values because of large $P_{T} \sim \mu$. The $n$-th term in the expansion reads

$$
\begin{aligned}
& \mathscr{A}^{(n)}=\delta\left(p_{1}^{+}+p_{2}^{+}-p_{0}^{+}\right) \int d^{2} b_{T} d^{2} r_{T} e^{-i\left(\vec{P}_{T} \cdot \vec{r}_{T}+\vec{k}_{T} \cdot \vec{b}_{T}\right)} \frac{r_{T}^{\mu} \Gamma_{\mu}}{r_{T}^{2}} \\
& \frac{1}{n !} r_{T}^{\alpha_{1}} \ldots r_{T}^{\alpha_{n}} \sum_{i=0}^{n}\left(\begin{array}{c}
n \\
i
\end{array}\right) \bar{z}^{i}(-z)^{n-i}\left(\partial_{\alpha_{1}} \ldots \partial_{\alpha_{i}} U^{R_{1}}\left(\vec{b}_{T}\right)\right) T^{R_{0}}\left(\partial_{\alpha_{i+1}} \ldots \partial_{n} U^{R_{2}}\left(\vec{b}_{T}\right)\right) .
\end{aligned}
$$

3. Isolation of 1-body contributions. We want to keep higher twist corrections, but not the multi-body corrections. Note that they are all mixed together in the CGC approach and the proper isolation requires integration by parts of the highly non-symmetric terms (we mean here the non-symmetry in derivatives acting on the first and the second Wilson line). The isolated 1-body contributions from the $n$-th term of the expansion reads

$$
\begin{aligned}
\mathscr{A}_{1-\mathrm{body}}^{(n)}= & \delta\left(p_{1}^{+}+p_{2}^{+}-p_{0}^{+}\right) \int d^{2} b_{T} d^{2} r_{T} e^{-i\left(\vec{P}_{T} \cdot \vec{r}_{T}+\vec{k}_{T} \cdot \vec{b}_{T}\right)} \frac{r_{T}^{\mu} \Gamma_{\mu}}{r_{T}^{2}} \\
& r_{T}^{\alpha} \sum_{j=0}^{n} \frac{\left(\overrightarrow{i k}_{T} \cdot \vec{r}_{T}\right)^{j}}{(j+1) !}\left\{\partial_{\alpha} U^{R_{1}}\left(\vec{b}_{T}\right) T^{R_{0}} U^{R_{2}}\left(\vec{b}_{T}\right)+U^{R_{1}}\left(\vec{b}_{T}\right) T^{R_{0}} \partial_{\alpha} U^{R_{2}}\left(\vec{b}_{T}\right)\right\} .
\end{aligned}
$$

4. Resummation and integration over $r_{T}$. The resummed one body contribution, containing thus all kinematic twists, reads

$$
\begin{aligned}
& \mathscr{A}_{1 \text {-body }}=\delta\left(p_{1}^{+}+p_{2}^{+}-p_{0}^{+}\right) \int d^{2} b_{T} e^{-i\left(\vec{k}_{T} \cdot \vec{b}_{T}\right)} \frac{\Gamma_{i}}{k_{T}^{2}}\left(k_{T}^{i} \delta^{j l}+k_{T}^{j} \delta i l-k_{T}^{l} \delta^{i j}\right) \\
& \left\{\left(\frac{P_{T}^{l}}{P_{T}^{2}}+\frac{p_{2 T}^{l}}{p_{2 T}^{2}}\right) \partial_{j} U^{R_{1}}\left(\vec{b}_{T}\right) T^{R_{0}} U^{R_{2}}\left(\vec{b}_{T}\right)+\left(\frac{P_{T}^{l}}{P_{T}^{2}}-\frac{p_{1 T}^{l}}{p_{1 T}^{2}}\right) U^{R_{1}}\left(\vec{b}_{T}\right) T^{R_{0}} \partial_{j} U^{R_{2}}\left(\vec{b}_{T}\right)\right\} .
\end{aligned}
$$

5. We pick up a concrete process (i.e. we fix the Dirac structure and the color representations), square the amplitude, average over color sources, and use the correspondence of the CGC color averages and hadronic matrix elements [3] (we also utilize the known fact that the derivative acting on the Wilson line extracts the gluon field). We find that the resulting expressions exactly match the ITMD expressions for different processes - see [7] for details. 


\section{Summary}

Although the CGC theory provides a complete framework for particle production in heavy ion collisions, certain components implicitly present in the calculations, complicate the actual phenomenology in some interesting cases, for instance for forward jet production at the LHC, which is potentially sensitive to saturation physics. We mean here notably the genuine MPI contributions (multipatron interactions) that should be suppressed for sufficiently hard jets. On the other hand, in the ITMD approach there are no higher body operators present and the formalism operates basing on the quite well known notion of the TMD parton distributions, with however nonlinear equations evolving them in $x$. In existing phenomenology studies, only approximate equations have been used (see [16] for initial works toward more complete treatment of the evolution).

In [7] we have performed the detailed derivation of the ITMD approach as the resummed kinematic twists to the one-body operators (at the amplitude level) in the CGC theory. As mentioned, phenomenologically, such approximation is reasonable for sufficiently hard jets (but still such that saturation scale is an important scale). In that regime, we must however take into account the Sudakov logarithms, which in real phenomenological studies have been done only approximately.

Thus, the future ambitious goals to achieve are: NLO corrections to hard off-shell gauge invariant hard factors and the complete set of solvable evolution equations together with the precise Sudakov resummation.

\section{References}

[1] Y.V. Kovchegov, Quantum Chromodynamics at High Energy, vol. 33, Cambridge University Press, 2012

[2] F. Gelis, E. Iancu, J. Jalilian-Marian and R. Venugopalan, Ann. Rev. Nucl. Part. Sci. 60, 463 (2010)

[3] F. Dominguez, C. Marquet, B. W. Xiao and F. Yuan, Phys. Rev. D 83, 105005 (2011)

[4] J. Collins, Foundations of perturbative $Q C D$, Cambridge Monographs on Particle Physics, Nuclear Physics and Cosmology 32, 1 (2011).

[5] T. Altinoluk, R. Boussarie, C. Marquet and P. Taels, JHEP 1907, 079 (2019)

[6] P. Kotko, K. Kutak, C. Marquet, E. Petreska, S. Sapeta and A. van Hameren, JHEP 1509, 106 (2015)

[7] T. Altinoluk, R. Boussarie and P. Kotko, JHEP 1905, 156 (2019)

[8] T. Altinoluk and R. Boussarie, JHEP 1910, 208 (2019)

[9] C. J. Bomhof, P. J. Mulders and F. Pijlman, Eur. Phys. J. C 47, 147 (2006)

[10] M. Bury, P. Kotko and K. Kutak, Eur. Phys. J. C 79, no. 2, 152 (2019)

[11] K. Kutak and S. Sapeta, Phys. Rev. D 86, 094043 (2012)

[12] A. van Hameren, P. Kotko, K. Kutak, C. Marquet, E. Petreska and S. Sapeta, JHEP 1612, 034 (2016) Erratum: [JHEP 1902, 158 (2019)]

[13] C. Marquet, E. Petreska and C. Roiesnel, JHEP 1610, 065 (2016)

[14] M. Aaboud et al. [ATLAS Collaboration], Phys. Rev. C 100, no. 3, 034903 (2019)

[15] A. van Hameren, P. Kotko, K. Kutak and S. Sapeta, Phys. Lett. B 795, 511 (2019)

[16] I. Balitsky and A. Tarasov, JHEP 1510, 017 (2015) , JHEP 1606, 164 (2016) 